rev.relac.int.estrateg.segur.7(1):173-189,2012

\title{
REZAGO SOCIAL Y DISCRIMINACIÓN DE LA POLÍTICA SOCIAL HACIA LOS GRUPOS INDÍGENAS EN SONORA*
}

Jorge E. Horbath** Amalia Gracia***

\section{RESUMEN}

Los procesos de discriminación y exclusión social hacia los indígenas en México han ido generando niveles de marginación y pobreza crecientes en los diversos colectivos. Aun con los esfuerzos realizados en materia de política social, los programas son insuficientes para cumplir con los derechos demandados por los pueblos indígenas pues no alcanzan a apuntalar una calidad de vida digna con garantías para sostener sus identidades y formas de organización social propias construidas colectivamente. Una mirada a la situación de los pueblos y comunidades indígenas en el Estado de Sonora, nos muestra un agigantado decrecimiento de su po-

* El documento es parte del proyecto "Exclusión, discriminación y pobreza de los indígenas urbanos en México. Similitudes y diferencias con otras experiencias latinoamericanas", seleccionado en la convocatoria 2008-2010 y financiado por el Fondo Sectorial para la Investigación en Educación SEP-CONACYT

** Investigador titular, Área Sociedad Cultura y Salud, El Colegio de la Frontera Sur (ECOSUR), Unidad Chetumal. Miembro del Sistema Nacional de Investigadores, S.N.I. Nivel 1 del CONACYT. jhorbath@ecosur.mx

*** Investigadora titular, Área Sociedad Cultura y Salud, El Colegio de la Frontera Sur (ECOSUR), Unidad Chetumal. Miembros del Sistema Nacional de Investigadores, S.N.I. Nivel 1 del CONACYT magracia@ecosur.mx 
blación respecto a la población no indígena; según cifras del Censo de Población de 2000 y el Conteo de Población de 2005, mientras los grupos de no indígenas habían aumentado en 9.07 por ciento, la población indígena decreció en 9.53 por ciento. En esta ponencia se analizan las condiciones de los indígenas en los municipios del Estado de Sonora, resaltando las condiciones de pobreza y la evolución de los componentes del rezago social, así como los aspectos de discriminación laboral que sufren en las ciudades.

Palabras clave: Exclusión social, indígenas urbanos, discriminación laboral, política social.

\title{
SOCIAL SETBACK AND DISCRIMINATION OF THE SOCIAL POLICIES TOWARD THE NATIVE GROUPS IN SONORA
}

\begin{abstract}
The practice of discrimination and social exclusion toward natives in Mexico have been generating levels of isolation and growing poverty in the various communities. Even with the efforts made in issues of social policy, programs are insufficient to fulfill the rights demanded by indigenous people, as they do not assure a worthy quality of life with guarantees to uphold their identities and their own collective forms of social organization. A look to the situation of the towns and native communities in the State of Sonora, shows a huge decrease of native vs. non-indigenous population; comparing figures of the 2000 and 2005 Census, while non-indigenous groups had increased by 9.07 per cent, the native population had decreased by 9.53 percent. This article analyzes the conditions of indigenous people in the municipalities of the State of Sonora, highlighting poverty conditions and the evolution of the components of the social setback, as well as aspects of the labor discrimination they suffer in the cities.
\end{abstract}

Key words: Social exclusion, urban natives, labor discrimination, social policy.

\section{ATRASO SOCIAL E DISCRIMINAÇÃO DA POLÍTICA SOCIAL PARA OS GRUPOS INDÍGENAS EM SONORA}

\section{RESUMO}

Os processos de discriminação e exclusão social dos indígenas no México vêm gerando um aumento nos níveis de marginalização e pobreza nos diversos coletivos. Apesar dos esforços realizados no campo da política social, os programas são insuficientes para cumprir os direitos reivindicados pelos povos indígenas, pois não conseguem fornecer uma qualidade de vida digna e garantir suas identidades e formas de organização social próprias, construídas coletivamente. Um olhar sobre a situação dos povos e comunidades indígenas no estado de Sonora mostra 
uma gigantesca redução da população em comparação com a população não indígena, de acordo com os dados do Censo de População de 2000 e a Contagem de População de 2005. Enquanto os grupos não indígenas aumentaram 9.07 por cento, a população indígena diminuiu 9.53 por cento. Neste artigo analisam-se as condições dos indígenas nos municípios do estado de Sonora, destacando as condições de pobreza, a evolução dos componentes do atraso social e também os aspectos da discriminação no trabalho que padecem as cidades.

Palavras chave: exclusão social, indígenas urbanos, discriminação no trabalho, política social.

\section{INTRODUCCIÓN}

Los procesos de discriminación y exclusión social hacia los indígenas en México han ido generando niveles de marginación y pobreza crecientes en los diversos colectivos. Aun con los esfuerzos realizados en materia de política social, los programas son insuficientes para cumplir con los derechos demandados por los pueblos indígenas pues no alcanzan a apuntalar una calidad de vida digna con garantías para sostener sus identidades y formas de organización social propias construidas colectivamente.

En esta ponencia se efectúa un recuento de la política social multicultural y el proceso que ha seguido la atracción del marco regulatorio internacional que le brinda garantías en materia de derechos a los pueblos y comunidades indígenas en México, dando cuenta de los diferentes instrumentos tanto internacionales como nacionales para ello, así como también la composición presupuestal dirigido hacia ese grupo poblacional. En el documento se analizan las condiciones de los indígenas en los municipios del Estado de Sonora, resaltando las condiciones de pobreza y la evolución de los componentes del rezago social, así como los aspectos de discriminación laboral que sufren en las ciudades como una manera de ilustrar los dramáticos niveles de desigualdad que sufren la población indígena en lo local, en un contexto territorial caracterizado por zonas desérticas y escasez de agua para nada favorable. Asimismo se revisan los principales programas sociales dirigidos a los grupos indígenas analizando sus insuficiencias en contextos urbanos en los que las necesidades de los grupos indígenas entran en tensión con la lógica del diseño e implementación de las políticas públicas.

\section{POLÍTICA SOCIAL MULTICULTURAL Y DERECHOS DE LOS PUEBLOS Y COMUNIDA- DES INDÍGENAS}

En primer lugar, se debe entender que la manera como se estructuró el Estado mexicano parte del proceso de conquista y colonización, entendido como la negación material y espiritual de un modo de vida, desarrollando una nueva construcción simbólica heredada del catolicismo, y una relación de dominadores y subordinados. Así pues, este proceso de conquista y coloniza- 
ción dejó repercusiones de largo alcance en la estructuración de la sociedad mexicana, primero desde este momento se construiría una línea racial que constituiría los lazos de dominación, a lo que se refiere Roux (2005:66) en una frontera definida por el color de la piel que quedaría inmersa junto con el destino de trabajo excedente, en la propia definición de lo que separaría a los oprimidos de las elites, y en términos de Fanon (1961:43) refiriéndose desde el contexto colonial, en la división del mundo, que es de entrada, el hecho de pertenecer o no a una determinada especie, y desde ahí, es de donde se construye la frontera racial entre dominadores y oprimidos, la elite blanca de un lado y la piel morena del otro; esto sería una de las herencias de mundo colonial en la sociedad mexicana que fundamenta a su vez lo que ha llegado a ser hasta hoy el Estado mexicano, a partir del eje racial, una línea divisoria, una fractura social entre dominantes y dominados.

De la colonización había quedado también el reconocimiento y la incorporación de las comunidades indígenas en la entidad política, evidentemente este proceso no dejó intactas las sociedades autóctonas, el mundo indígena fue cristalizado, castellanizado y obligado a adaptar sus antiguas formas de producción comunal para fines que les eran ajenos. El resultado fue un híbrido, o como lo llama Echevarria (1998), un proceso de mestizaje cultural en el cual tuvieron que rehacerse, reestructurarse y reconstruirse mutuamente para poder integrarse.

La revolución y otros conflictos posrevolucionarios que atravesaron todo el siglo XIX, tuvieron que ver con la reconfiguración interna de la sociedad mexicana y una nueva reordenación del Estado posterior a la reorganización del conflicto armado. La disputa jurídica sobre el artículo 27 y en particular sobre la propiedad nacional de la tierra y el petróleo fue una de sus expresiones. De este periodo sociopolítico, quedo promulgada la constitución de 1917, el Estado mexicano se convertía así en precursor de lo que seria las siguientes décadas de un proceso mundial de recomposición estatal fundado en la deformación del derecho público y en el reconocimiento y negociación de las clases subalternas organizadas como fuente de legitimidad estatal.

Las instituciones básicas de respaldo a las garantías sociales (gobierno, mercado, familias) se desgastan peligrosa y simultáneamente sin poder descargar entre sí las responsabilidades que ya no pueden satisfacer algunas o todas. Más aún, las mejoras parciales recientes en las condiciones de pobreza obedecen más a los esfuerzos y sacrificios adaptativos de la población -ocupaciones múltiples, trabajo femenino, migración y remesas-que a los efectos de las políticas públicas.

La crónica crisis fiscal del Estado impide que los órganos gubernamentales asuman funciones sociales en escala suficiente; la prestación de servicios sociales vía el mercado excluye al grueso de los hogares pobres o de ingresos bajos; las familias -y singularmente las mujeres- absorben el costo de la transición económica, pero sus capacidades menguan ante el embate de la escasez de empleos, los bajos ingresos y los complejos fenómenos sociodemográficos que disuelven los núcleos familiares. 
La parte central del problema deriva de la incongruencia entre las estrategias microsociales incluyentes y los enfoques macroeconómicos excluyentes, incapaces en conjunto de atacar con verdadera efectividad los problemas de la marginación y la pobreza. El sector moderno de la economía, sobre todo las actividades industriales, ha dejado de absorber a las oleadas generacionales de nuevos trabajadores y de emplear los excedentes de mano de obra de la agricultura. Es decir, se ha creado un mecanismo macroeconómico y macrosocial perverso de fomento a la exclusión que los programas microsociales alivian pero no son capaces de erradicar.

La situación descrita se viene traduciendo en el crecimiento explosivo del sector informal, en pobreza crónica, En rigor, ganar la batalla contra la injusticia social y los rezagos económicos implica dar un contundente golpe de timón a la orientación de las políticas públicas en varios frentes, incluida la necesidad de hacer paulatinamente exigibles los programas micro-sociales, así como negar aprobación a reformas que, por sus efectos primarios o secundarios, alienten la exclusión y los sesgos concentradores del ingreso nacional.

\section{INSTRUMENTOS EN EL TEMA DE DERECHOS DE LOS PUEBLOS INDÍGENAS.}

\subsection{INSTRUMENTOS INTERNACIONALES}

Uno de los principales instrumentos internacionales de carácter vinculante orientado a la protección de derechos de los pueblos indígenas es el Convenio 169 sobre los Pueblos Indígenas y Tribales en Países Independientes de la Organización Internacional del Trabajo (OIT) de 1989, aprobado por el Congreso Mexicano en 1990, entrando en vigor en el ámbito nacional un año después.

La importancia de este Convenio radica en la introducción y reconocimiento de los indígenas como pueblo, la definición de los mismos a partir de las características históricas, y el desarrollo de temas entorno a derechos sociales y culturales no asumidos en declaraciones o convenciones precedentes. En ese sentido, ha constituido el principal instrumento de exigencia de derechos por parte de la población indígena en todo el mundo. De igual forma, se reconoce a este Convenio el cambio de perspectiva en el tratamiento acerca de las libertades y derechos de los pueblos indígenas, puesto que se aleja de la visión integracionista instalada en la acción de los Estados durante años ${ }^{1}$.

Por oposición a este enfoque, el Convenio 169 deja claro a los Estados la obligación de incluir a los pueblos indígenas en la formulación de las acciones gubernamentales, relacionada con la

1. Puede consultarse el Convenio 107 sobre poblaciones indígenas y tribales de 1957. 
coordinación y protección de sus propios derechos en materia de tierras, condiciones de empleo, salud, educación y formación profesional. Lo anterior, por medio de la creación de instancias de participación específicas que les permitan a éstos incorporarse en los procesos de decisión gubernamental que les conciernan o afecten, ya sean de carácter legislativo o administrativo. Sin embargo, a pesar de los alcances en cuanto al reconocimiento de derechos que esta Convención contiene, en todo su articulado no reivindica de modo explícito derechos políticos, en términos de libre autodeterminación y autonomía, razón por la cual ha sido objeto de crítica por parte de algunos sectores entre los que se encuentran la academia y las mismas organizaciones de indígenas. El argumento más citado que sustenta dicha crítica es la aclaración que se hace al respecto de la noción "pueblo", en un sentido diferente y no comparable con el asignado a los Estados-nación en el derecho internacional ${ }^{2}$, en tanto se considera, limita la toma de decisiones y accionar de los pueblos indígenas a los ámbitos que discrecionalmente les asigna el Estado.

Muy recientemente, esta inconformidad quedó por lo menos jurídicamente saldada, con la Declaración de las Naciones Unidas sobre los Derechos de los Pueblos Indígenas del 13 de septiembre de 2007, un nuevo instrumento normativo internacional, que fue aprobado luego de más de veinte años de consultas y de foros internacionales entre Estados y organizaciones de la sociedad civil entre las que por supuesto se incluyen las indígenas.

Sin ningún tipo de restricción, esta Declaración avanza en el tema de la autonomía, estableciendo en el artículo tres (3) que "Los pueblos indígenas tienen derecho a la libre determinación. En virtud de ese derecho determinan libremente su condición política y persiguen libremente su desarrollo económico, social y cultural".

Con este nuevo esquema, se les concede oficialmente a los pueblos indígenas el carácter de sujetos políticos -más allá de estrictas reivindicaciones sociales o culturales- con todo lo que esto implica y en concordancia con el Pacto Internacional de Derechos Civiles y Políticos y el Pacto Internacional de Derechos Económicos, Sociales y Culturales, ambos adoptados en 1966 y entrados en vigor diez años después.

Al respecto, la Declaración propone la participación activa de los pueblos indígenas en la construcción de políticas públicas (artículo 23 ONU, Declaración sobre los derechos de los pueblos indígenas, 2007). Esto supone un cambio en las relaciones entre la población indígena y el Estado, a partir de un nuevo tratamiento a la población indígena, que pasa de ser simple receptora de las políticas y programas del gobierno, a ser partícipes en la construcción de alternativas

2. Al respecto, el artículo uno (1) numeral tres (3) declara "la utilización del término "pueblos" en este Convenio no deberá interpretarse en el sentido de que tenga implicación alguna en lo que atañe a los derechos que pueda conferirse a dicho término en el derecho internacional". 
para darle solución a sus problemas y necesidades más sentidas. Propósito que se hará efectivo en la medida en que los Estados "dejen de pensar por esta población" y creen mecanismos de participación idóneos, que superen las instancias de consulta en que los encierran.

Entre otros derechos reivindicados en la Declaración, se encuentran: el derecho y respeto por los sistemas de tenencia y goce de la tierra que habita la población indígena desde tiempos pasados; el derecho de implementar su propio sistema jurídico; el derecho a establecer sus propios medios de información en sus propios idiomas; la igualdad de género entre otros. Como siempre, está en manos de cada Estado adoptar acciones legislativas y administrativas encaminadas a hacer de los ideales de esta Declaración una realidad, en ese sentido es preciso explorar las posibilidades que hasta ahora ofrece la normatividad nacional.

\subsection{INSTRUMENTOS NACIONALES}

En la normatividad nacional dirigida a proteger los derechos de los pueblos indígenas se encuentra la Ley General de Derechos Lingüísticos de los Pueblos Indígenas del 13 de marzo de 2003, cuyo objetivo principal es la protección de los derechos lingüísticos, individuales y colectivos de los pueblos indígenas, que poseen su propia lengua antes de la conformación del Estado Mexicano. Siguiendo este propósito se crea el Instituto Nacional de Lenguas Indígenas (INALI) que será el encargado a través de una acción descentralizada de promover y preservar las lenguas indígenas en toda la Federación. En la práctica, esta disposición es significativa puesto que las lenguas indígenas adquieren legalmente la misma validez y vigencia que el español, abriendo la posibilidad a sus hablantes para comunicarse y relacionarse sin restricciones en cualquier espacio en que se desenvuelvan, sin limitar su práctica al entorno comunitario.

Las consecuencias de estas disposiciones son importantes sobre todo en el tema educativo y jurídico. En cuanto al primero, se adecuó la Ley General de Educación para que estuviera en correspondencia con el espíritu de esta nueva normatividad, reformando el artículo séptimo (70), fracción IV, de la Ley General de Educación de 2003, en la cual se compromete al sector educativo en la promoción de la pluralidad lingüística y la enseñanza obligatoria en lengua indígena a la población hablante de la misma.

Por otra parte, en el tema jurídico se implementan en el Código Federal de Procedimientos Civiles $^{3}$ dos cambios fundamentales, el primero consiste en la obligatoriedad de asistir en diligencias y procesos legales con intérpretes, traductores y defensores a la población indígena, en el caso en que ésta lo requiera, esto es, cuando no se comunique en español y cuando carezca de un defensor que le asesore. El segundo cambio consiste en la consideración de los

3. Consultar reformas y adiciones de los artículos 6o;15;18;124 bis;128 fracción IV; $154 ; 159$ y 220 bis, del 18 de diciembre de 2002. Y la modificación del artículo 85 en el año 2003. 
usos y costumbres indígenas en el momento de formular y tomar decisiones judiciales como sanciones y penas.

Cabe anotar que si bien estos dos cambios en materia judicial resultan favorables para el respeto de los derechos de la población indígena, se ha evidenciado que en la práctica no se llevan a cabo como está estipulado en las normas y los trámites ante las instancias judiciales se hacen más lentos y tortuosos. Un ejemplo de esto consiste en la dificultad, tanto para las instituciones como para población indígena, en la obtención de traductores que los asista en los procesos judiciales. Entre las causas que generan esta situación está el déficit de traductores o intérpretes, entre otras cosas por problemas de pago por la prestación de sus servicios.

El segundo instrumento normativo de la legislación nacional es la Ley de la Comisión Nacional para el Desarrollo de los Pueblos Indígenas de 2003, por medio de la cual se crea la Comisión Nacional para el Desarrollo los Pueblos Indígenas (CDI), sustituyendo al Instituto Nacional Indigenista (INI) que funcionó desde 1948 como el responsable a nivel federal de todas las políticas públicas concernientes a la población indígena. A diferencia del esquema centralizado de intervención del INI, la nueva Comisión se propuso como un organismo descentralizado y autónomo en materia técnica, administrativa y presupuestal, que a través de un trabajo transversal e integral está encargada de la orientación, coordinación institucional, promoción, seguimiento y evaluación todas las políticas públicas dirigidas a la población indígena.

La CDI se involucra directamente con la salvaguarda de los derechos de los indígenas en tanto que por ley, no sólo debe proponer alternativas, sino que también debe coordinar, colaborar y evaluar la acción de las diferentes entidades de la Administración Pública Federal que desarroIlan programas dirigidos a dicha población. De otro lado, la CDI debe hacer el seguimiento a las acciones de todas las entidades que desarrollan acciones y programas específicos en este tema, por eso tiene la obligación de elaborar y publicar de forma anual un informe sobre el estado de avance e impacto logrado con las mismas. Sin embargo, pese a todas las acciones por encadenar la ley y su cumplimiento, sigue apreciándose un evidente rezago en materia de necesidades frente a acciones efectivas dirigidas a la construcción de garantías que acerquen a la población indígena a formas de inclusión en los distintos ámbitos de la construcción social.

\section{EL PRESUPUESTO EN MATERIA INDÍGENA EN MÉXICO}

Según el informe de "Análisis del presupuesto en materia indígena: 2001-2009" de Violeta Núñez ${ }^{4}$ realiza un análisis de los datos sobre presupuesto gubernamental en materia indígena

4. Violeta R. Núñez Rodríguez (2009) "Análisis del presupuesto en materia indígena: 2001-2009", Centro de Estudios para el Desarrollo Rural Sustentable y la Soberanía Alimentaria. Cámara de Diputados, LX Legislatura. 
durante el sexenio 2001-2006 y durante la presente administración gubernamental. Entre algunos de los resultados de éste, tenemos los siguientes:

1. El presupuesto del Instituto Nacional Indigenista (INI) y la Comisión Nacional para el Desarrollo de los Pueblos Indígenas (CDI) se incrementó en términos reales, del primer año en relación con el último del sexenio, en un 172 por ciento. El crecimiento promedio del periodo fue de 18 por ciento.

2. Durante los dos primeros años, los recursos fueron enfocados en promover el desarrollo en localidades y grupos marginados o en situación de pobreza, desigualdad y exclusión.

3. En la primera etapa de la administración -todavía con el INI en funciones-, los programas a los que más recursos se destinaron fueron Fondos Regionales y Albergues Escolares.

4. A partir de la creación de la CDI, el Programa de Infraestructura Básica para la Atención de los Pueblos Indígenas (PIBAI), comenzó a ser el programa con la mayor cantidad de presupuesto. Para el año 2004, se dirigió a él, más de la mitad de los recursos destinados a la CDI; en 2005, esta proporción alcanzó el 65\%; y para 2006, implicó un poco más de la mitad del presupuesto total.

5. La principal estrategia gubernamental desde la creación de la CDI, fue la de dotar de infraestructura básica a pueblos y comunidades indígenas. En fechas recientes la Estrategia 100×100 también ha tenido un papel protagónico, debido a que al abarcar los municipios con menor Índice de Desarrollo Humano, coincide con las regiones con mayor presencia indígena $^{5}$.

6. En cuanto al presupuesto total para atender a la población indígena (no sólo el de la CDI), éste tuvo un crecimiento real de $66 \%$ (del primer año en relación con el último), y un crecimiento promedio de 9\%, según el 6 Informe de Gobierno. Por su parte, según la CDI, el crecimiento fue de $157 \%$, y el promedio de 17 por ciento.

7. Es difícil conocer cómo se ejercen los recursos en materia indígena, debido a que el Decreto de PEF no desglosa el total de las erogaciones en la materia, ${ }^{6}$ sólo menciona escasos rubros dirigidos a la atención de estos pueblos y comunidades.

8. Para el año 2003, únicamente el PEF desglosó 2,468 millones de pesos; es decir, 14\% de las erogaciones en materia indígena. En 2004, los recursos desglosados ascendieron a

5. Para 2008, las dependencias federales involucradas en esta Estrategia comprometieron una inversión de 6,300 millones de pesos (www.microregiones.gob.mx). En este mismo año, como lo veremos en las páginas siguientes, a toda la CDI se dirigió un poco más de siete mil millones de pesos.

6. A partir del año 2008 se realizó el desglose en el PEC. 
4,561 millones de pesos, 23\% del total. En 2005, sólo se conoció el destino de una tercera parte. Y en el último año del sexenio, del total del presupuesto, sólo fue posible saber el destino del 25 por ciento.

9. Con base en los datos de los informes de la CDI (única fuente en donde se realiza un ejercicio de desglose del presupuesto en materia indígena, basado en criterios territoriales), ${ }^{7}$ las secretarías y dependencias gubernamentales a las que más recursos se destinaron durante el sexenio, fueron: la Secretaría de Educación Pública, Secretaría de Desarrollo Social, CDI, Secretaría de Agricultura, Ganadería, Desarrollo Rural, Pesca y Alimentación y la Secretaría de Salud. A éstas (incluido el Ramo 33) se dirigió más del 80\% del presupuesto.

10. En los últimos tres años del sexenio, la SEP ocupó, por mucho, el primer lugar de destino del gasto. En promedio, se llevó el $40 \%$ de los recursos totales en materia indígena.

11. En cuanto al número de programas gubernamentales, en la primera mitad del sexenio, el presupuesto indígena se dirigió a 78 programas de diferentes secretarías y dependencias. De los cuales 46 involucraban a la SEP, SEDESOL, CDI y SSA.

12. En cuanto a recursos, la SEDESOL fue la secretaría que en los primeros tres años se llevó, alrededor de una tercera parte del presupuesto. Desarrollo Humano Oportunidades fue el programa que contó con más recursos.

13. A partir de la creación de la CDI (en la segunda mitad del sexenio), el presupuesto en materia indígena comenzó a tener otro comportamiento. El número de programas involucrados fue aproximadamente de $104^{8}$. El programa de Educación Básica Preescolar

7. La CDI en su Informe 2006, indica que ante la carencia de una identificación de la variable indígena en la mayoría de los programas federales, se diseñaron criterios territoriales para la identificación y validación del presupuesto por parte de las dependencias y organismos de la APF, "lo cual dio como resultado la definición de un conjunto de 871 municipios indígenas..." (Acciones de Gobierno para el Desarrollo Integral de los Pueblos Indígenas. Informe 2006, CDI, México, 2007). Al respecto, en Indicadores socioeconómicos de los pueblos indígenas, publicado en 2002 por el INI Programa de las Naciones Unidas para el Desarrollo, los 871 municipios son aquellos: "en donde la estimación de población indígena (PI) era mayor al 40\%, los cuales se consideraron eminentemente indígenas" (655 municipios). Asimismo, se identificaron dos grupos más: “... aquellos en los que la PI presenta un volumen igual o mayor a 5,000 personas, los cuales se consideran de interés debido a que cuentan con presencia absoluta de población indígena y aquellos municipios con presencia de población que habla alguna lengua con menos de 5,000 habitantes" (216 municipios).

8. Un estudio realizado por el CONEVAL en 2007 —Diagnóstico de las políticas públicas federales para la población indígena en México (1990-2007) — identifica, a lo largo de estos diecisiete años, entre 149 y 160 estrategias, políticas, y programas federales (la misma fuente presenta estos dos datos) que han atendido y atienden a la población indígena. En este trabajo sólo se presentan las que estuvieron vigentes en el sexenio anterior (2000-2006) y en lo que va del actual (2007-2009). 
y Primaria Indígena (de la SEP), ocupó un lugar protagónico en cuanto al destino del presupuesto (ya no el de Oportunidades). En promedio tuvo alrededor del $26 \%$ de los recursos.

\section{LAS CIFRAS DEL REZAGO SOCIAL INDÍGENA EN SONORA.}

De acuerdo al Informe de Desarrollo Humano de los Pueblos Indígenas de México 2006 (IDH 2006:78,79), que formula el índice de desarrollo humano a través de tres componentes: la educación, medida por el grado de alfabetización de adultos mayores de 15 años y la asistencia escolar de 6 a 24 años; la salud medida por la sobrevivencia infantil; y el ingreso en relación al consumo. La población indígena presenta grandes diferencias con respecto a la población no indígena, en temas como la educación, la salud, y sobre todo los ingresos. Aclarando que dentro de los diferentes Estados de México se presentan algunas diferencias entre el IDH de la población indígena, a nivel nacional se encuentra que: El IDH de los pueblos indígenas es de 0.7057, frente al IDH de la población no indígena, que es de 0.8304, encontrándose una diferencia de aproximadamente $15 \%$. En cuanto al índice de salud, la población indígena alcanza un 0.7380 , frente a un índice de 0.8491 para los no indígenas, esto quiere decir que existe una diferencia de 13.1 por ciento, entre ambas poblaciones.

En el tema de educación, se encuentran las diferencias más grandes entre la población indígena y no indígena. En primer lugar, esto se evidencia porque el índice de educación de los pueblos indígenas es de 0.7319 , frente a un 0.8841 de la población no indígena. Existiendo una brecha de 17.2 por ciento, entre ambas poblaciones. En segundo lugar, a esta condición contribuye la baja asistencia escolar entre la población indígena en edad escolar, cuyo índice de asistencia es de 0.7392 , frente a un índice de 0.8029 de la población no indígena. En tercer lugar, la diferencia entre el índice de alfabetismo entre ambas poblaciones indígena y no indígena, supera el 21 por ciento. La población indígena tiene un índice de alfabetismo de 0.7283, mientras que la población no indígena alcanza un índice de 0.9247 por ciento.

En el tema de los ingresos, también existe una diferencia notable, para la población indígena el índice de ingresos es de 0.6471 y para los no indígenas es de 0.7579 , esto es una brecha de 14,6 por ciento. En relación con la vivienda, los ingresos son importantes porque posibilitan o no el acceso a la misma en condiciones dignas y seguras.

Según el estudio "Cambios en la situación nutricional de México 1990- 2000" a través del Índice de Riesgo Nutricional elaborado por el Instituto Nacional de Ciencias Medicas y Nutrición Salvador Zubirán y la Sociedad Latinoamericana de Nutrición (2003), en la zona norte occidental de México es donde encontraron prácticamente la única área crítica de desnutrición del "México del norte". Una franja que corre a lo largo de la Sierra Madre Occidental, con dos pequeñas regiones que resaltan: la primera al noroeste de la república donde coinciden los límites de los estados de Sonora, Sinaloa y Chihuahua, que es donde se localizan a los grupos 
indígenas Mayos, Yaquis y Tarahumaras; y la segunda siguiendo la sierra occidental hacia el sur, donde se encuentran las fronteras de Durango, Nayarit, Zacatecas y Jalisco, donde ubicamos los grupos indígenas tepehuanos, Coras y Huicholes.

Por su proximidad con la frontera norte de México, su cercanía entre ambas ciudades y su diversidad socio-productiva, las ciudades de Guaymas y Hermosillo en el Estado de Sonora se convierten en excelente referencia para aportar en el análisis del fenómeno de la discriminación y exclusión social de los indígenas en las ciudades mexicanas.

De acuerdo a los datos arrojados por el censo de 2000, la población total de Hermosillo era de 609.829 habitantes, de los cuales 12.592 eran indígenas, representando a 2.1 por ciento de la población total. De ellos, 96.9 por ciento eran bilingües con primera lengua mixteca y segunda lengua náhuatl. Por sectores de actividad económica, la población indígena activa se distribuye un 30.5 por ciento (\%) en el sector primario, 23.4 por ciento (\%) en el secundario y 46 por ciento (\%) en el terciario. Las viviendas que habitan los indígenas en la ciudad de Hermosillo muestra condiciones precarias según los datos del censo 2000, pues 26.3 por ciento (\%) tienen piso de tierra, cerca de 17 por ciento (\%) no disponen de agua entubada, 10.2 por ciento (\%) no disponen de servicio sanitario ni excusado, más de 7 por ciento no cuentan con energía eléctrica y más de 16 por ciento (\%) cocinan con leña o carbón.

En Guaymas, según datos del censo de 2000 la población total era de 130.329 habitantes, con una población indígena de 15.417 habitantes, representando un 11.8 por ciento de la población. Los indígenas que reportaron se bilingües representaban a 92.3 por ciento del total de población indígena de Guaymas, siendo la lengua yaqui su primera lengua, mientras que la segunda era la náhuatl. Su distribución por actividades productivas mostraba que el 46.3 por ciento (\%) se vinculaba al sector primario, 32.8 por ciento (\%) al sector secundario y el restante 20.9 por ciento (\%) al sector terciario. En Guaymas las condiciones precarias de los indígenas son mayores que las registradas en Hermosillo, pues 63.9 por ciento (\%) de las viviendas que habitan tienen piso de tierra, 12.2 por ciento (\%) no disponen de agua entubada, 26.7 por ciento no cuentan con sanitario ni excusado, 17.2 por ciento (\%) no tienen energía eléctrica y 57.7 por ciento (\%) cocinan con leña o carbón.

Una mirada a la situación de los pueblos y comunidades indígenas en el Estado de Sonora, nos muestra un agigantado decrecimiento de su población respecto a la población no indígena; según cifras del Censo de Población de 2000 y el Conteo de Población de 2005, mientras los grupos de no indígenas habían aumentado en 9.07 por ciento, la población indígena decreció en 9.53 por ciento. Los informes gubernamentales sobre la pobreza indican que la población indígena ha venido reduciendo su rezago social principalmente por el efecto de los programas sociales. Sin embargo, en 21 de los 31 municipios que tienen importante presencia de población indígena, se registraron fuertes contracciones pues la población se ve obligada a migrar, principalmente a las ciudades. Esto cuestiona la efectividad de los programas sociales que no 
garantizan el derecho a permanecer en el territorio con lo cual además exponen a los indígenas a nuevos procesos de discriminación y segregación que aun más difícilmente atenderán.

Al observar con detenimiento los valores resultantes del índice de rezago social para la población indígena de los municipios de Sonora estimado por la CDI-PNUD y calcular su crecimiento porcentual durante 2000-2005, se tiene que el índice se redujo 14.6 por ciento en un lapso de cinco años (ver tabla 2). Por tipo de municipio no se logra apreciar que exista evidencia estadística que pueda dar una asociación entre la dispersión o concentración de población indígena y la variación del índice, debido a que en una parte importante de los municipios (9 de 31 municipios observados) se registró una reducción del rezago social de los indígenas, pero entre los municipios que muestran un aumento del rezago, se caracterizan por tener una población indígena dispersa.

Tabla 1. Municipios de Sonora: Crecimiento de la población indígena y no indígena entre 2000 y 2005.

\begin{tabular}{|c|c|c|c|c|c|c|c|c|c|c|}
\hline Entidad/Municipio & $\begin{array}{l}\text { Total } \\
2000\end{array}$ & \begin{tabular}{|c|}
$\begin{array}{c}(\%) \\
\text { Indige na }^{2} \\
2000\end{array}$ \\
\end{tabular} & \begin{tabular}{|c|}
$\begin{array}{c}(\%) \\
\text { Indigena }\end{array}$ \\
2000
\end{tabular} & $\begin{array}{l}\text { Total } \\
2005\end{array}$ & $\begin{array}{c}(\%) \\
\text { Indigena }^{2} \\
2005\end{array}$ & \begin{tabular}{|c}
$\begin{array}{c}\text { (\%) No } \\
\text { Indigena } \\
2005\end{array}$ \\
\end{tabular} & $\begin{array}{l}\text { Total } \\
\text { Crec. \% } \\
\text { 00-05 }\end{array}$ & \begin{tabular}{|c|} 
Indíge na \\
Crec. $\%$ \\
$00-05$
\end{tabular} & \begin{tabular}{|c|} 
No \\
Indigena \\
Crec. $\%$ \\
$00-05$ \\
\end{tabular} & Tipo de municipio \\
\hline Nacional Indígena & $97,483,412$ & 10.48 & 89.52 & $98,465,647$ & 10.01 & 89.99 & 1.01 & -3.59 & 1.55 & \\
\hline SONORA & $2,216,969$ & 5.61 & 94.39 & $2,394,861$ & 4.70 & 95.30 & 8.02 & -9.53 & 9.07 & \\
\hline 002 AGUA PRIETA & 61,944 & 2.13 & 97.87 & 70,303 & 1.23 & 98.77 & 13.49 & -34.44 & 14.54 & Con PI dispersa \\
\hline 012 BÁCUM & 21,322 & 12.71 & 87.29 & 20,892 & 10.71 & 89.29 & -2.02 & -17.45 & 0.23 & Con PI dispersa \\
\hline 017 CABORCA & 69,516 & 2.42 & 97.58 & 70,113 & 1.90 & 98.10 & 0.86 & -20.61 & 1.39 & Con presencia indígena \\
\hline 018 CAJEME & 356,290 & 2.31 & 97.69 & 375,800 & 1.83 & 98.17 & 5.48 & -16.47 & 5.99 & Con presencia indigena \\
\hline 019 CANANEA & 32,061 & 0.97 & 99.03 & 32,157 & 0.78 & 99.22 & 0.30 & -19.29 & 0.49 & Con PI dispersa \\
\hline 025 EMPALME & 49,987 & 2.36 & 97.64 & 50,663 & 1.64 & 98.36 & 1.35 & -29.69 & 2.10 & Con Pl dispersa \\
\hline 026 ЕTCHOJOA & 56,129 & 42.23 & 57.77 & 55,697 & 38.35 & 61.65 & -0.77 & -9.89 & 5.90 & Indigena \\
\hline 027 FRONTERAS & 7,801 & 3.28 & 96.72 & 7,470 & 2.85 & 97.15 & -4.24 & -16.80 & -3.82 & Con PI dispersa \\
\hline 029 GUAYMAS & 130,329 & 11.64 & 88.36 & 134,153 & 11.40 & 88.60 & 2.93 & 0.88 & 3.20 & Con presencia indigena \\
\hline 033 HUATABAMPO & 76,296 & 24.69 & 75.31 & 74,533 & 21.98 & 78.02 & -2.31 & -13.04 & 1.21 & Con presencia indigena \\
\hline 035 IMURIS & 9,988 & 1.17 & 98.83 & 10,517 & 1.84 & 98.16 & 5.30 & 65.81 & 4.58 & Con PI dispersa \\
\hline 036 MAGDALENA & 24,447 & 0.75 & 99.25 & 25,524 & 0.60 & 99.40 & 4.41 & -15.85 & 4.56 & Con Pl dispersa \\
\hline 039 NACO & 5,370 & 1.97 & 98.03 & 6,010 & 2.26 & 97.74 & 11.92 & 28.30 & 11.59 & Con PI dispersa \\
\hline 041 NACOZARI DE GARCÍA & 14,365 & 2.65 & 97.35 & 11,961 & 2.96 & 97.04 & -16.74 & -6.84 & -17.00 & Con PI dispersa \\
\hline 042 NAVOJOA & 140,650 & 14.75 & 85.25 & 144,598 & 11.89 & 88.11 & 2.81 & -17.12 & 6.25 & Con presencia indígena \\
\hline 043 NOGALES & 159,787 & 2.46 & 97.54 & 193,517 & 1.86 & 98.14 & 21.11 & -8.34 & 21.85 & Con PI dispersa \\
\hline 047 PITIQUITO & 9,236 & 3.68 & 96.32 & 9,117 & 4.50 & 95.50 & -1.29 & 20.59 & -2.12 & Con presencia indígena \\
\hline 048 PUERTO PEÑASCO & 31,157 & 2.60 & 97.40 & 44,875 & 3.01 & 96.99 & 44.03 & 66.34 & 43.43 & Con $\mathrm{PI}$ dispersa \\
\hline 049 QUIRIEGO & 3,335 & 10.94 & 89.06 & 3,049 & 8.30 & 91.70 & -8.58 & -30.68 & -5.86 & Con PI dispersa \\
\hline 055 SAN LUIS RÍO COLORADO & 145,006 & 1.55 & 98.45 & 157,076 & 1.00 & 99.00 & 8.32 & -30.28 & 8.93 & Con presencia indígena \\
\hline 056 SAN MIGUEI DE HORCASITAS & 5,626 & 17.97 & 82.03 & 6,036 & 27.20 & 72.80 & 7.29 & 62.41 & -4.79 & Con PI dispersa \\
\hline 058 SANTA ANA & 13,526 & 0.95 & 99.05 & 14,638 & 1.01 & 98.99 & 8.22 & 14.73 & 8.16 & Con PI dispersa \\
\hline 069 YÉCORA & 6,069 & 10.48 & 89.52 & 6,089 & 11.25 & 88.75 & 0.33 & 7.70 & -0.53 & Con presencia indigena \\
\hline 070 GENERAL PLUTARCO ELÍAS CAL & 11,278 & 3.52 & 96.48 & 12,416 & 2.05 & 97.95 & 10.09 & -36.02 & 11.77 & Con presencia indigena \\
\hline 071 BENTTO JUÁREZ & 21,813 & 13.34 & 86.66 & 20,447 & 13.45 & 86.55 & -6.26 & -5.43 & -6.39 & Con PI dispersa \\
\hline O72 SAN IGNACIO RIOO MUERTO & 13,692 & 14.26 & 85.74 & 13,244 & 16.37 & 83.63 & -3.27 & 11.07 & -5.66 & Con Pl dispersa \\
\hline
\end{tabular}

${ }_{1}^{1}$ Municipios con 100 o más habitantes indígenas.

${ }^{2}$ Excluye a la población indígena en viviendas sin información de ocupantes.

Nota: Los índices generados toman valores positivos y negativos alrededor de su media que es de cero, debido a esto, el índice es transformado a una escala de cero a cien, con lo cual se puede expresar el rezago social de las unidades geográficas en términos porcentuales, así comparar y ubicar la posición en la que se encuentran respecto a las demás.

Fuente: CDI-PNUD. Sistema de Indicadores sobre la población indigena en México con base en: INEGI, II Conteo de Población y Vivienda, México, 2005. 
Respecto a cada uno de los cuatro componentes del índice, en el rezago educativo (compuesto por \% de la población analfabeta de 15 años y más, \% de la población que no asiste a la escuela de 6 a 14 años y \% de la población sin instrucción o sin primaria completa de 15 años y más), el rezago aumentó en una buena parte de los municipios de Sonora, lo que agudiza el proceso de discriminación y de exclusión en espacios productivos. Mientras que para el índice de rezago en infraestructura social básica (\% de ocupantes en viviendas particulares sin agua entubada, \% de ocupantes en viviendas particulares sin drenaje y \% de ocupantes en viviendas particulares sin energía eléctrica), se redujo solamente en 2 por ciento en toda la entidad y aumentó en 11 de los 31 municipios se registró un aumento del rezago en infraestructura.

Tabla 2. Municipios de Sonora: Crecimiento del índice de rezago social y sus componentes para la población indígena y no indígena entre 2000 y 2005.

\begin{tabular}{|c|c|c|c|c|c|c|c|c|c|c|c|c|c|c|c|c|}
\hline \multirow[b]{2}{*}{ Entidad/M unicipio } & \multirow[b]{2}{*}{ Tipo de municipio } & \multicolumn{3}{|c|}{$\begin{array}{c}\text { Índice de Rezago Social } \\
\text { de los Pueblos } \\
\text { Indigenas (IRSPI) }\end{array}$} & \multicolumn{3}{|c|}{$\begin{array}{c}\text { Índice de Rezago } \\
\text { Educativo }\end{array}$} & \multicolumn{3}{|c|}{$\begin{array}{c}\text { Indice de Rezago en } \\
\text { Infraestructura } \\
\text { Social Básica } \\
\end{array}$} & \multicolumn{3}{|c|}{\begin{tabular}{|c|} 
Índice de Rezago en \\
Condiciones de la \\
Vivienda \\
\end{tabular}} & \multicolumn{3}{|c|}{\begin{tabular}{|c|}
$\begin{array}{c}\text { Indice de Rezago en } \\
\text { Bienes Dentro de la } \\
\text { Vivienda }\end{array}$ \\
\end{tabular}} \\
\hline & & 2000 & 2005 & \begin{tabular}{|c|} 
Crec. $\%$ \\
$00-05$
\end{tabular} & 2000 & 2005 & \begin{tabular}{|c|} 
Crec. $\%$ \\
$00-05$
\end{tabular} & 2000 & 2005 & \begin{tabular}{|c|} 
Crec. \% \\
$00-05$
\end{tabular} & 2000 & 2005 & \begin{tabular}{|c|} 
Crec. $\%$ \\
$00-05$
\end{tabular} & 2000 & 2005 & \begin{tabular}{|c|} 
Crec. $\%$ \\
$00-05$
\end{tabular} \\
\hline
\end{tabular}

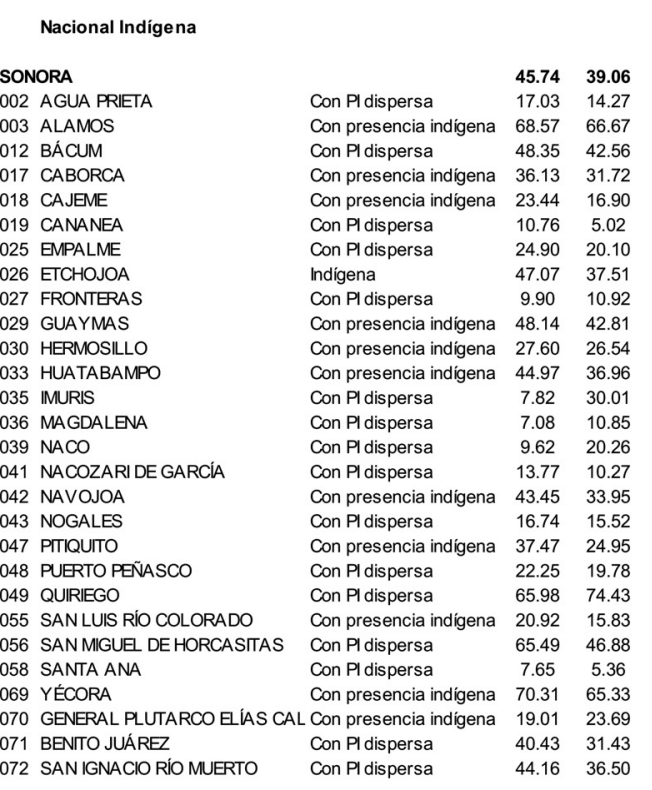

$\begin{array}{rcc}-14.60 & 26.86 & 29.58 \\ -16.17 & 9.09 & 10.68 \\ -2.78 & 43.97 & 38.37 \\ -11.98 & 27.92 & 25.24 \\ -12.19 & 29.27 & 26.16 \\ -27.90 & 16.23 & 12.61 \\ -53.35 & 14.85 & 6.06 \\ -19.27 & 14.94 & 14.28 \\ -20.32 & 22.22 & 18.70 \\ 10.32 & 5.04 & 3.69 \\ -11.07 & 26.41 & 22.28 \\ -3.85 & 21.14 & 21.80 \\ -17.82 & 22.82 & 20.30 \\ 283.57 & 5.03 & 37.34 \\ 53.23 & 5.11 & 14.48 \\ 110.66 & 11.15 & 13.89 \\ -25.40 & 12.82 & 8.97 \\ -21.85 & 22.47 & 19.97 \\ -7.30 & 6.86 & 7.01 \\ -33.40 & 22.18 & 21.25 \\ -11.12 & 18.09 & 15.25 \\ 12.80 & 44.91 & 49.13 \\ -24.36 & 17.52 & 15.63 \\ -28.41 & 64.74 & 48.24 \\ -29.91 & 4.13 & 4.91 \\ -7.08 & 47.92 & 42.53 \\ 24.65 & 14.63 & 12.84 \\ -22.27 & 26.29 & 19.46 \\ -17.35 & 24.17 & 19.15\end{array}$

\begin{tabular}{rcc}
10.12 & 36.57 & 35.83 \\
17.50 & 6.25 & 7.01 \\
-12.76 & 55.14 & 56.34 \\
-9.62 & 25.28 & 20.92 \\
-10.62 & 23.54 & 19.94 \\
-22.32 & 10.15 & 6.76 \\
-59.21 & 3.26 & 2.24 \\
-4.42 & 17.44 & 12.37 \\
-15.84 & 29.87 & 24.62 \\
-26.84 & 3.57 & 0.60 \\
-15.65 & 28.54 & 25.96 \\
3.12 & 14.58 & 14.53 \\
-11.07 & 30.78 & 26.22 \\
642.40 & 1.68 & 7.22 \\
183.08 & 1.97 & 6.51 \\
24.62 & 5.87 & 9.10 \\
-30.03 & 1.67 & 2.00 \\
-11.12 & 29.60 & 23.25 \\
2.21 & 16.58 & 15.41 \\
-4.20 & 38.14 & 11.74 \\
-15.70 & 11.37 & 8.59 \\
9.40 & 38.74 & 64.28 \\
-10.77 & 14.54 & 7.21 \\
-25.48 & 24.05 & 7.94 \\
18.83 & 1.04 & 1.28 \\
-11.24 & 54.28 & 55.88 \\
-12.26 & 16.69 & 21.17 \\
-25.98 & 22.20 & 18.62 \\
-20.77 & 27.11 & 27.44 \\
& & \\
\hline
\end{tabular}

\begin{tabular}{rcc}
$\mathbf{- 2 . 0 2}$ & $\mathbf{4 1 . 6 3}$ & 44.05 \\
12.31 & 16.50 & 14.94 \\
2.18 & 54.53 & 61.56 \\
-17.24 & 53.13 & 53.34 \\
-15.28 & 32.05 & 32.42 \\
-33.36 & 23.34 & 19.03 \\
-31.11 & 6.84 & 2.51 \\
-29.08 & 19.84 & 19.89 \\
-17.57 & 42.53 & 36.93 \\
-83.13 & 7.68 & 8.35 \\
-9.04 & 49.15 & 50.94 \\
-0.35 & 26.01 & 28.03 \\
-14.79 & 37.07 & 34.47 \\
329.53 & 10.69 & 30.25 \\
229.82 & 12.24 & 4.30 \\
54.91 & 11.78 & 27.30 \\
19.36 & 13.68 & 11.71 \\
-21.45 & 40.14 & 34.24 \\
-7.06 & 18.02 & 17.22 \\
-69.22 & 18.45 & 14.11 \\
-24.51 & 21.22 & 22.27 \\
65.92 & 56.47 & 68.96 \\
-50.45 & 20.37 & 15.30 \\
-66.99 & 63.61 & 53.16 \\
22.22 & 8.72 & 2.50 \\
2.95 & 52.41 & 46.18 \\
26.82 & 14.68 & 14.62 \\
-16.15 & 31.49 & 27.75 \\
1.18 & 43.52 & 38.37 \\
& & \\
\hline
\end{tabular}

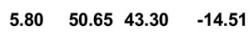
$\begin{array}{llll}-9.46 & 30.17 & 19.21 & -36.31\end{array}$ $\begin{array}{llll}12.89 & 84.30 & 82.35 & -2.31\end{array}$ $\begin{array}{lllll}0.40 & 61.31 & 53.49 & -12.76\end{array}$ $\begin{array}{llll}1.17 & 41.39 & 30.99 & -25.12\end{array}$ $\begin{array}{llll}-18.46 & 31.55 & 21.22 & -32.73\end{array}$ $\begin{array}{llll}-63.27 & 12.62 & 6.79 & -46.19\end{array}$ $\begin{array}{llll}0.27 & 33.20 & 23.47 & -29.32\end{array}$ $\begin{array}{llll}-13.17 & 67.28 & 52.21 & -22.40\end{array}$ $\begin{array}{llll}8.67 & 19.23 & 27.61 & 43.60\end{array}$ $\begin{array}{llll}3.64 & 62.56 & 53.18 & -15.00\end{array}$ $\begin{array}{llll}7.79 & 35.36 & 29.83 & -15.63\end{array}$ $\begin{array}{llll}-7.02 & 63.82 & 48.81 & -23.51\end{array}$ $\begin{array}{llll}182.94 & 9.58 & 30.90 & 222.50\end{array}$ $\begin{array}{llll}-64.85 & 6.89 & 11.80 & 71.18\end{array}$ $\begin{array}{llll}131.64 & 4.44 & 23.87 & 437.48\end{array}$ $\begin{array}{llll}-14.39 & 18.19 & 13.23 & -27.30\end{array}$ $\begin{array}{llll}-14.70 & 57.35 & 41.19 & -28.18\end{array}$ $\begin{array}{llll}-4.45 & 21.14 & 18.29 & -13.50\end{array}$ $\begin{array}{llll}-23.52 & 54.61 & 40.50 & -25.83\end{array}$ $\begin{array}{llll}4.94 & 25.78 & 23.29 & -9.64\end{array}$ $22.12 \quad 88.4486 .40 \quad-230$ $\begin{array}{llll}-24.90 & 21.57 & 16.73 & -22.42\end{array}$ $\begin{array}{llll}-16.42 & 74.13 & 48.97 & -33.94\end{array}$ $\begin{array}{llll}-71.33 & 12.11 & 9.48 & -21.72\end{array}$ $\begin{array}{llll}-11.87 & 88.76 & 86.09 & -3.01\end{array}$ $\begin{array}{llll}-0.35 & 21.45 & 37.28 & 73.77\end{array}$ $\begin{array}{llll}-11.86 & 58.08 & 44.40 & -23.56\end{array}$ $\begin{array}{llll}-11.84 & 57.86 & 44.69 & -22.77\end{array}$

\section{${ }^{1}$ Municipios con 100 o más habitantes indigenas.
${ }^{2}$ Excluye a la población indigena en viviendas sin información de ocupantes.}

Nota: Los índices generados toman valores positivos y negativos alrededor de su media que es de cero, debido a esto, el índice es transformado a una escala de cero a cien, con lo cual se puede expresar el rezago social de las unidades geográficas en términos porcentuales, así comparar y ubicar la posición en la que se encuentran respecto a las demás.

Fuente: CDI-PNUD. Sistema de Indicadores sobre la población indigena en México con base en: INEGI, II Conteo de Población y Vivienda, México, 2005.

En el componente de rezago en condiciones de la vivienda (\% de ocupantes en viviendas particulares con piso de tierra, \% de ocupantes en viviendas particulares sin sanitario exclusivo, $\%$ de 
ocupantes en viviendas particulares en los que más de dos personas duermen en un cuarto), sufrió un incremento de 5.8 por ciento, siendo evidente el aumento en 11 de los 31 municipios observados. En tanto que en el componente de bienes dentro de la vivienda (\% de ocupantes en viviendas particulares sin televisión, \% de ocupantes en viviendas particulares sin refrigerador y $\%$ de ocupantes en viviendas particulares sin lavadora), se logró una reducción de 14.5 por ciento entre 2000 y 2005, aumentando solamente en 5 municipios.

La observación y reflexión de los componentes del rezago social entre la población indígena muestra la huella del alcance de la política pública en Sonora, concentrada en infraestructura y en bienes a los integrantes de los hogares, especialmente a la población infantil, producto de los programas de lucha contra la pobreza como el programa Oportunidades. Sin embargo, los esfuerzos en materia educativa todavía no se reflejan en el componente y por el contrario, si se considera lo que ocurre con los programas de educación básica y la alta deserción escolar entre los niños y jóvenes indígenas, se evidencia la necesidad de un ajuste oportuno. Lo mismo ocurre con el componente de condiciones de la vivienda, pues también pasa por el acceso a recursos que permitan su mejoramiento, pero considerando la identidad y cultura en el hábitat que los indígenas han construido en sus comunidades.

\section{CONCLUSIONES}

La política pública en los tres niveles de gobierno se desarrolla de manera transversal. No obstante cada una de las entidades de la APF, como se anotó antes, no realiza la formulación y ejecución de los programas con base en una previa diferenciación de la población por atender en dichos programas, y por lo tanto, no se planean programas específicos para la población indígena a partir de sus particularidades étnicas, culturales, sociales y políticas. Por otra parte, en Sonora como en el resto del país, la mayoría de los programas que abordan los temas de educación, salud y vivienda que nos interesa analizar, no están formulados para población indígena, sino para población no indígena. En ese sentido, no se están aplican criterios de discriminación positiva dentro de los mismos programas, obviando así, los parámetros que se suponen deben guiar una política social, como se anotó en páginas anteriores. Sumado a esto, desde el lado estatal y municipal en Sonora no se tiene claridad por parte de las entidades sobre la población indígena que es atendida por dichos programas, y su referencia de atención a esta población es geográfica.

Si bien existen espacios de consulta a los pueblos indígenas sobre temas que son problemas para ellos, en la entidad no existe una participación efectiva por parte de los mismos en la toma de decisiones de política pública. Situación que de acuerdo al concepto de política pública, deslegitima en parte el proceso de construcción de las mismas. Esto a pesar, de los instrumentos internacionales que se han proferido para que los pueblos indígenas participen de manera autónoma y organizada en la formulación y diseño de los programas en temas cruciales para su desarrollo. 
El cambio de situación dependerá igualmente de la creación de espacios y mecanismos de participación para las organizaciones indígenas, así como para los indígenas no organizados, además de un tratamiento por parte del Estado, que parta del reconocimiento de la población indígena como sujeto político y no objeto de política. Así se podrá tener una política pública incluyente que no reproduzca prácticas discriminatorias.

La normatividad actual en materia indígena, que deviene de los acuerdos internacionales firmados por México y las demás naciones, han mostrado ser insuficientes como leyes para hacer que la desigualdad y la persistencia en la discriminación, con su consecuente permanencia en los históricos márgenes de pobreza, puedan ser reducidos de manera significativa en México. Las instituciones y su asignación presupuestal, así como el diseño de política pública, lejos de lograr cambios significativos, construyen falsos peldaños de apoyo que generan dependencia de las comunidades indígenas pobres de tales recursos, al retirarse tales peldaños vuelven a la misma condición en que estaban. Dicha articulación entre normatividad y política pública debe diseñarse enfocada en endogeneizar el desarrollo local y articular lo local al desarrollo regional. Tal ruptura hace que se prologue la pobreza y estimule el deterioro social en las comunidades indígenas.

\section{BIBLIOGRAFÍA}

- Cazés, Daniel (1980), "Zapotecas rebeldes rechazan ser indios profesionales", en El Día, Publicaciones Mexicanas, México, 1 de julio, p. 19.

- $\quad$ CDI (2009), Índice de Rezago Social (IRSPI) 2000 - 2005, Comisión Nacional para el Desarrollo de los Pueblos Indígenas. México, D.F.

- CDI-PNUD (2005), Sistema de Indicadores sobre la Población Indígena en México. MéxiCO, D.F.

- Coneval, (2007), "Diagnóstico de las políticas públicas federales para la población indígena en México (1990-2007)", Consejo Nacional de Evaluación de la Política de Desarrollo Social, México, D.F.

- Echevarría, Bolívar (1988), "Valor de uso y utopía". Siglo Veintiuno Editores, México

- Fanon, Frantz, (1961), "les damnés e la terre", Paris: la découverte.

- Instituto Nacional de Ciencias Medicas y Nutrición Salvador Zubirán y la Sociedad Latinoamericana de Nutrición (2003), Cambios en la situación nutricional de México 19902000" a través del Índice de Riesgo Nutricional. 
- INEGI (2011), XIII Censo de Población y Vivienda de México, Resultados definitivos. México, D.F. INEGI, [en línea] www.inegi.gob.mx/censos2010.

- $\quad$ Núñez Rodríguez, Violeta R. (2009), “Análisis del presupuesto en materia indígena: 20012009", Centro de Estudios para el Desarrollo Rural Sustentable y la Soberanía Alimentaria. Cámara de Diputados, LX Legislatura.

- $\quad$ PNUD (2006), Informe de desarrollo humano de los pueblos indígenas de México. MéxiCO, D.F.

- Roux, Rinha, (2005), “El príncipe mexicano. Subalternad, historia y Estado". Ediciones Era: México.

- Sánchez, Consuelo (1999), "Los pueblos indígenas, del indigenismo a la autonomía". Siglo XXI Editores. México.

- Sánchez, Consuelo (1999), "La construcción del sujeto político. Los pueblos indígenas, del indigenismo a la autonomía", México: Siglo XXI editores.

- Warman, Arturo (2003), "Los indios mexicanos en el umbral del milenio", México: FCE. 